Research Article

\title{
An Improved Equation for Predicting Compressive Stress in Posttensioned Anchorage Zone
}

\author{
Young-Ha Park, ${ }^{1}$ Moon-Young Kim $\mathbb{D D}^{2},{ }^{2}$ Jong-Myen Park, ${ }^{1}$ and Se-Jin Jeon $\mathbb{D D}^{3}$ \\ ${ }^{1}$ Jiseung C\&I, Seoul 06120, Republic of Korea \\ ${ }^{2}$ School of Civil and Architectural Engineering, Sungkyunkwan University, Suwon-si 16419, Republic of Korea \\ ${ }^{3}$ Department of Civil Systems Engineering, Ajou University, Suwon-si 16499, Republic of Korea
}

Correspondence should be addressed to Se-Jin Jeon; conc@ajou.ac.kr

Received 23 July 2019; Accepted 29 January 2020; Published 28 February 2020

Academic Editor: Stefano de Miranda

Copyright (c) 2020 Young-Ha Park et al. This is an open access article distributed under the Creative Commons Attribution License, which permits unrestricted use, distribution, and reproduction in any medium, provided the original work is properly cited.

\begin{abstract}
Validity of the approximate equation for predicting compressive stress in the posttensioned anchorage zone presented in the AASHTO LRFD Bridge Design Specifications was investigated in this study. Numerical analysis based on the finite element method (FEM) and theoretical analysis showed that the AASHTO formula gives relatively accurate stress values when the effect of duct holes is neglected. However, it was found that the formula can significantly overestimate the stresses in the actual prestressed concrete member with spaces occupied by ducts. Therefore, an improved equation was proposed for the existing AASHTO equation to consider the effect of the duct holes on the stress distribution. This resulted in relatively accurate prediction of the distribution and magnitude of the compressive stresses even with the presence of the duct holes. The proposed equation was also validated by comparing with the stresses measured in the test of a posttensioned full-scale specimen. This study is expected to contribute to the design of the anchorage zone in prestressed concrete structures by suggesting a more reasonable way to assess the appropriateness of anchorage devices.
\end{abstract}

\section{Introduction}

The prestressing force of a prestressing tendon is transmitted to a concrete section by an anchorage device, inducing compressive stress in posttensioned prestressed concrete (PSC) structures [1]. The prestressing force concentrated on the anchorage device spreads as it proceeds to the interior of the member and the compressive stress is almost uniformly distributed throughout the cross-section when it reaches a certain depth. The anchorage zone is the volume of concrete through which the concentrated prestressing force at the anchorage device spreads as a constant stress distribution across the entire cross-section at a certain distance from the anchorage device. This zone should be designed based on the theories of disturbed region because of the complexity in stress distribution.

For the design of the anchorage zone, the following design methods can be used [2]: (1) equilibrium-based inelastic model (strut-and-tie model); (2) refined elastic stress analyses; or (3) other approximate methods using predictive equations. The design concept of the anchorage zone given by AASHTO has been adopted in many countries including Korea [3].

In the previous studies, reasonable strut-and-tie models for the analysis of the anchorage zone were suggested [4] and incorporated in provisions [2]. Also, efforts have been made to improve the predictive equations provided for bursting force among several design equations for the anchorage zone presented in AASHTO [5-7]. On the other hand, a large portion of the previous studies focused on improving the details of end-region reinforcements that can effectively control the cracks [8-13]. Investigation on the stress distribution at the anchorage zone is also important when developing new types of anchorage devices provided for the tendons made of advanced materials such as fiber reinforced polymer (FRP) [14-16]. However, relatively few studies have 
been performed on the improvement of the predictive equation for compressive stress in the special anchorage device. Even in the study that described the background of AASHTO LRFD recommendations for the design of the local anchorage zone [11], the equation for compressive stress was not dealt with.

In this study, the validity of the simplified approximate method in (3) was investigated among the above three methods presented in the AASHTO LRFD Bridge Design Specifications [2]. In particular, the background of the AASHTO equation for calculating the internal compressive stress was revisited among several predictive equations used in the approximate method. The validity of this equation was analytically examined by comparison with the results of finite element (FE) analyses. As a result, it was found that the original AASHTO equation can significantly overestimate the stresses in an actual PSC structure with spaces occupied by ducts. Therefore, an improved equation was proposed in this study that can yield more accurate stresses in the anchorage zone. The proposed equation was validated by comparing with the stresses obtained from FE analyses and with those measured in the test of a posttensioned full-scale specimen.

\section{Predictive Equations for Anchorage Zone Design}

In order to cope with any harmful situation anticipated in the anchorage zone shown in Figure 1(a), the simplified approximate calculation method presented in the AASHTO LRFD Bridge Design Specifications [2] provides equations for predicting the magnitude and location of bursting forces and spalling forces associated with tensile stresses and cracks that can occur in the anchorage zone. It is specified that the reinforcements against these forces should be arranged. In addition, in the case of a special anchorage device [11] that does not satisfy the specifications for bearing stress, the compressive stress induced inside the anchorage zone should be calculated and should be kept below the allowable value according to equation (1) in terms of load-and-resistance factor design (LRFD).

Such a compressive stress is important in the design and selection of an anchorage device because it can be affected by the specification, size, number, and spacing of the anchorage device. Although several conditions are required in terms of the configuration of the anchorage zone for applying this equation [2], including the condition of a rectangular crosssection, many of the PSC structures such as a PSC girder can be examined through this equation because they satisfy these conditions:

$$
f_{c a}=\frac{0.6 P_{u} \kappa}{A_{b}\left[1+l_{c}\left(\left(1 / b_{\text {eff }}\right)-(1 / t)\right)\right]} \leq 0.7 \phi f_{c i}^{\prime},
$$

where (refer to Figure 1(b)) $f_{c a}$ is concrete compressive stress at a distance of $l_{c}$ from the surface of an anchorage device $(\mathrm{MPa}), P_{u}$ is factored prestressing force of a tendon corresponding to each anchorage device $(\mathrm{N}), \kappa$ is correction factor for closely spaced anchorages, $A_{b}$ is effective bearing area of anchor plate in which the area of the duct should be deducted $\left(\mathrm{mm}^{2}\right), l_{c}$ is longitudinal extent of confining reinforcement of the local zone, but not more than the larger of $1.15 a_{\text {eff }}$ or $1.15 b_{\text {eff }}(\mathrm{mm}), a_{\text {eff }}$ is lateral dimension of the effective bearing area measured parallel to the larger crosssection dimension $(\mathrm{mm}), b_{\text {eff }}$ is lateral dimension of the effective bearing area measured parallel to the smaller crosssection dimension $(\mathrm{mm}), t$ is member thickness at the anchorage zone $(\mathrm{mm}), \phi$ is resistance factor (also called strength reduction factor), and $f_{c i}^{\prime}$ is specified compressive strength of concrete at time of prestressing $(\mathrm{MPa})$.

Equation (1) is a formula for predicting the concrete compressive stress at the boundary between the local zone and the general zone immediately outside the local zone at the anchorage zone where a special anchorage device is used. The local zone is defined as the rectangular or equivalent rectangular prism of the concrete surrounding and immediately ahead of the anchorage device and any integral confining reinforcement, while the general zone can be assumed to be identical to the overall anchorage zone including the local zone. $\kappa$ is introduced to consider the overlapped region of concrete stress for the multiple anchorages arranged in vertical direction in Figure 1(b) and is a function of the center-to-center spacing of anchorages $(s)$, number of anchorages in a row $(n)$, and $a_{\text {eff }} \cdot a_{\text {eff }}$ and $b_{\text {eff }}$ are the lateral dimensions of an anchor plate but can be increased in the case of a larger bearing area of the confined concrete than the anchor plate or can be decreased for an anchor plate which is not sufficiently stiff [2]. In the context of this study, vertical direction means the direction where the anchorages are arranged in a row as is typical of a PSC girder shown in Figure 1(b), whereas transverse direction indicates the through-thickness direction.

Equation (1) was adopted in the AASHTO LRFD Bridge Design Specifications based on previous studies [17, 18]. It was derived by multiplying the longitudinal stress distribution in the vertical direction with that in the transverse direction, for a typical anchorage zone of PSC structures, as shown in Figure 1. Therefore, although equation (1) is the formula that deals with three-dimensional (3D) stress distribution, the effect of the structural behavior in each direction on the stress distribution was considered separately. In detail, the magnitude of the compressive stress of the struts was determined as $0.6 f_{b}$ in the vertical direction through the analyses using the strut-and-tie model [4] for one anchorage, in which various angles of the struts were examined. Here, $f_{b}$ is the bearing stress which is applied at an anchor plate supporting the anchor head where a number of prestressing strands are anchored and can be calculated using $f_{b}=P_{u} / A_{b}$. On the other hand, the distribution of longitudinal stress in the transverse direction was obtained by assuming that $f_{b}$ spreads along a certain path, such as that indicated by the dotted lines in Figure 2 which corresponds to the plan view of Figure 1(b), resulting in an almost constant stress distribution throughout the crosssection at the depth of $t$ according to Saint-Venant's principle [19]. Therefore, the longitudinal stress at an arbitrary distance $x$ can be obtained, as shown in Figure 2, if $x$ is substituted for $l_{c}$ in equation (1), then equation (1) 


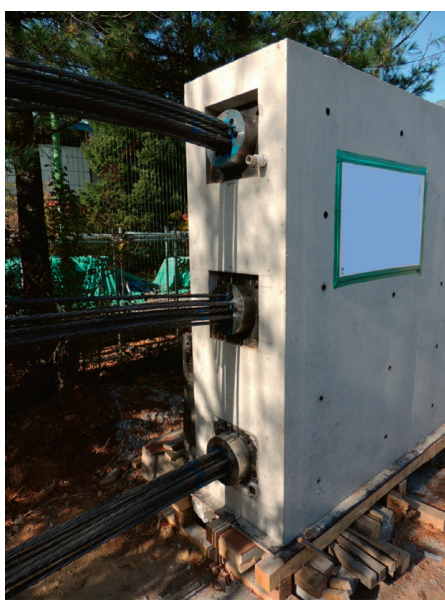

(a)

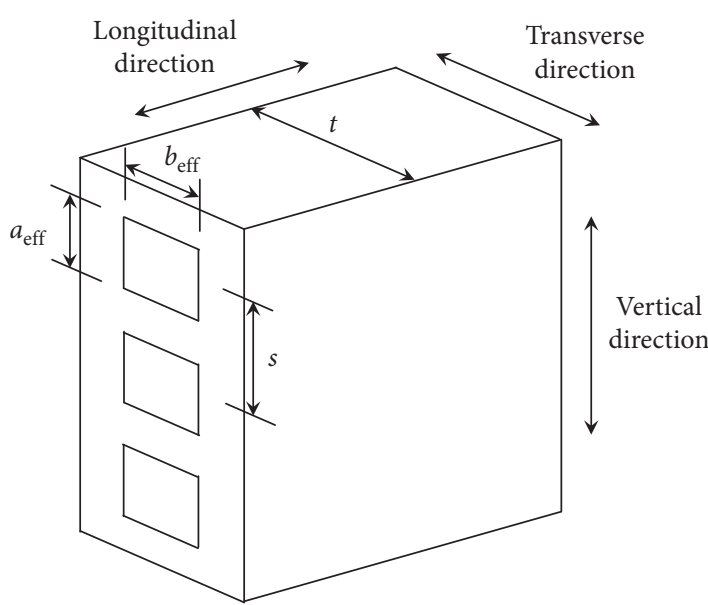

(b)

Figure 1: Anchorages of a PSC girder: (a) anchorages before tensioning; (b) notations.

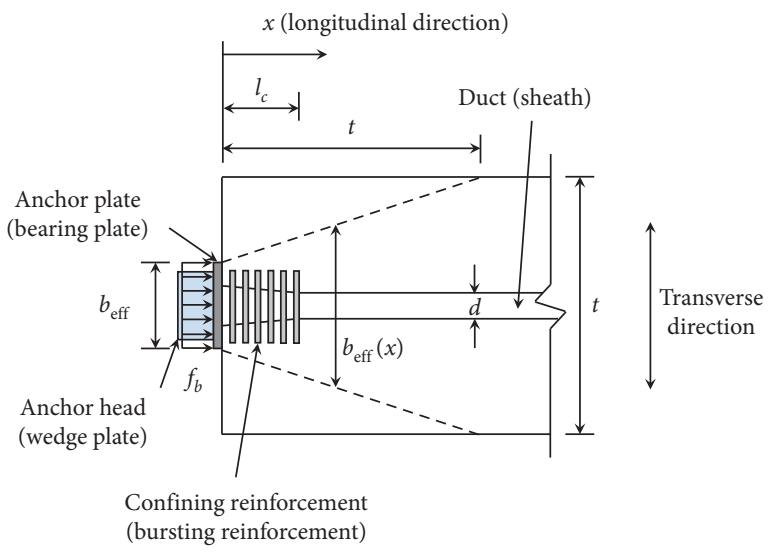

FIGURE 2: Longitudinal stress distribution in anchorage zone (plan view of Figure 1(b)).

corresponds to the stress at $x=l_{c}$. Although Figure 2 shows that the stress spreads linearly, the validity of this assumption of the linearity will be revisited later. Referring to Figure 2, the force applied to an anchor head in which a certain number of prestressing strands are wedged is transferred to the anchor plate. Figure 3 shows the inside of a PSC girder before casting the concrete, indicating the anchorage device with the back side of the anchor plate, the duct, and the confining reinforcement arranged in a spiral shape.

Equation (1) has been used in its originally proposed form without significant modification because few studies have been performed on the improvement of this equation. Therefore, the validity of the approximation made in equation (1) was analytically investigated in this study to determine whether it can accurately predict the stress distribution in the transverse direction in an actual configuration of an anchorage zone. As mentioned earlier, equation (1) was derived from relevant previous studies $[17,18]$. In the

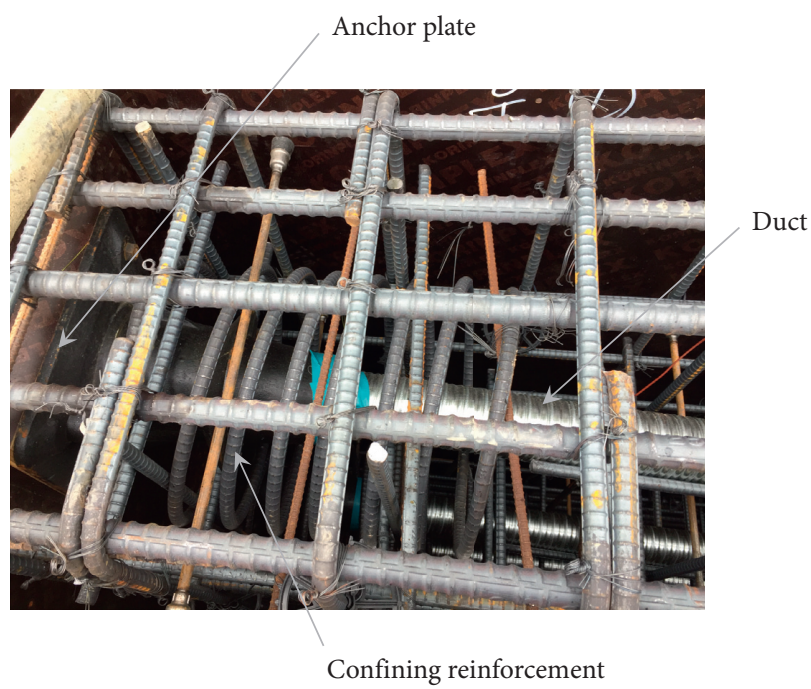

FIgURE 3: Anchorage device, duct, and confining reinforcement.

earliest study [18], the deduction of the duct area when calculating $A_{b}$ was not discussed and the depth for checking the compressive stress is specified as not $l_{c}$ but $a_{\text {eff }}$ or $b_{\text {eff }}$. However, in the subsequent NCHRP Report 356 [17] based on the study by Burdet [18], the deduction of the duct area in $A_{b}$ was additionally specified, and the depth for stress checking was revised to $l_{c}$ with the upper limit of $1.15 a_{\text {eff }}$ or $1.15 b_{\text {eff }}$. These provisions of the NCHRP Report 356 were adopted in the AASHTO LRFD Bridge Design Specifications and have been maintained without further modification.

However, if the effect of the duct hole is taken into account, the compressive stresses calculated using equation (1) significantly differ from those obtained using a more accurate methodology such as FE analysis. The reasons for this difference and possible improvement are investigated in the following sections. 


\section{Comparison between Design Equation and FE Analysis of Anchorage Zone}

3.1. General Remarks. The two-dimensional (2D) problem shown in Figure 2, which had been used to derive equation (1) in the study by Burdet [18], was analyzed by FE analysis and the results were compared with those obtained from equation (1) in this study. In general, 3D analysis would be required to examine the stress distribution in the disturbed or discontinuity region of anchorage zone in detail. However, because this study aims to verify the form of equation (1) especially in terms of spreading behavior of the longitudinal stress, 2D analysis would be adequate for this purpose. That is, as was described in detail in chapter 1 , because $0.6 \kappa$ of equation (1) represents the characteristics of the stress distribution in the vertical direction, the remaining part of equation (1) which is of interest in this study can be examined by $2 \mathrm{D}$ analysis. More detailed FE analyses, including material nonlinearity and modeling of the reinforcements such as confining reinforcement, are not crucial in this study, because the spreading phenomenon of concrete stress expressed in equation (1) is based on the linear elastic approach of homogeneous material prior to ultimate stage. It can be noted that the distribution of compressive stresses which is the main concern of this study is not much affected by the stress-strain relationship of concrete and by the presence of reinforcements.

During the analyses, the validity of equation (1) was examined in detail by considering two cases: an anchorage zone with a duct hole and an anchorage zone without a duct hole. A general-purpose FE analysis program, ABAQUS [20], was used and a plane stress element, CPS4R, was adopted among various $2 \mathrm{D}$ elements available in ABAQUS. CPS4R refers to a quadrilateral element with four nodes at its corners and with a reduced integration scheme. In order to sufficiently cover the region of constant stress, the longitudinal length of the analysis model of anchorage zone was determined as two times the member thickness. Through the preliminary analyses using various sizes of elements, the finite element mesh was selected to be sufficiently fine for the stress to converge to an accurate value. Each element is square-shaped with a side length of $10 \mathrm{~mm}$ and a thickness of $1 \mathrm{~mm}$. For the boundary conditions, roller supports and hinge supports were selectively used at appropriate locations to avoid restraining the free deformation, similar to an actual situation, as shown later.

On the other hand, although the location in which equation (1) is applied depends on the larger of $l_{c}, 1.15 a_{\text {eff }}$, and $1.15 b_{\text {eff }}$, the stress at $x=1.15 b_{\text {eff }}$ was consistently examined in this study assuming the usual case of $a_{\text {eff }}=b_{\text {eff }}$. While one of the reasons for this approach is the difficulty in determining a specific value of $l_{c}$ which depends on the design of confining reinforcements, the main reason is that the location at which the stress is examined is not significant because the purpose of this study is to compare the values obtained by FE analysis and by the design equation. Also, although some additional specifications are used in determining the effective bearing area as mentioned previously, the effective bearing area was regarded identical to the actual area of an anchor plate for the same aforementioned reason; therefore, $a_{\text {eff }}$ and $b_{\text {eff }}$ correspond to the actual sizes of the anchor plate.

3.2. Case without a Duct Hole. The configuration of the case without a duct hole is shown in Figure 4 and was modeled for the FE analyses. Note that the support conditions were applied to all the relevant nodes continuously although they were indicated at discrete locations in Figure 4 only for an illustrative purpose. In these boundary conditions, the right edge can deform in the transverse direction with the center fixed. The modulus of elasticity of $E_{c}=3 \times 10^{4} \mathrm{MPa}$ and Poisson's ratio of $\nu=0.17$ were used, assuming a certain ordinary concrete herein. However, these assumed values of $E_{c}$ and $v$ have a negligible effect on the resulting stresses in the plane stress or plane strain problems without a body force as used in this study. This is because the governing equation has the form of equation (2), in which the terms are not related to $E_{c}$ and $\nu$ [19]:

$$
\left(\frac{\partial^{2}}{\partial x^{2}}+\frac{\partial^{2}}{\partial y^{2}}\right)\left(\sigma_{x x}+\sigma_{y y}\right)=0,
$$

where $\sigma_{x x}$ and $\sigma_{y y}$ are the stresses in the $x$ and $y$ directions, respectively. In the following analyses, $b_{\text {eff }}$ was set to $300 \mathrm{~mm}$ and $t$ was varied to 429,600 , and $1,000 \mathrm{~mm}$ for the $b_{\text {eff }} / t$ of $0.7,0.5$, and 0.3 , respectively. However, the results of these analyses not only are effective for the specific dimensions and loads assumed herein, but also cover more general cases, the reason of which is as follows: equation (1) can be converted to equation (3) by introducing the longitudinal ( $x$-directional) stress $f_{b}(x)$ at an arbitrary location $x$ instead of $f_{c a}$ at a specific location. Note that the variable $x$ was used in equation (3) instead of $l_{c}$ in equation (1) and $f_{b}=P_{u} / A_{b}$ :

$$
\frac{f_{b}(x)}{P_{u} / A_{b}}=\frac{f_{b}(x)}{f_{b}}=\frac{0.6 \kappa}{1+(x / t)\left(\left(1 /\left(b_{\mathrm{eff}} / t\right)\right)-1\right)} .
$$

Therefore, examination of the validity of equation (1) is the same as that of equation (3), which includes the nondimensional variables of $f_{b}(x) / f_{b}, x / t$, and $b_{\text {eff }} / t$. In other words, the value of $b_{\text {eff }} / t$ rather than the separate values of $b_{\text {eff }}$ and $t$ has an effect on the stress ratio $f_{b}(x) / f_{b}$, and the range of $b_{\text {eff }} / t=0.3 \sim 0.7$ examined in this study falls within the possibility of practical application. On the other hand, $f_{b}$ was assumed to be unity ( $1 \mathrm{MPa})$ to facilitate the analysis, because $f_{b}(x)$ is then equal to $f_{b}(x) / f_{b}$.

When the prestressing strands are anchored, the FE analysis shows that the distribution of $f_{b}(x)$ is not constant at an arbitrary section corresponding to $x$, given that $x<t$. In detail, the distribution of the $x$-directional stresses at $x$ along the $y$ direction is depicted in Figure 5. The main concern of this distribution is the maximum value expressed by $f_{b, \max }(x)$ which occurs at the midsection $(y=0)$. Then, if the curved distribution of the stress is converted to an equivalent rectangular stress block with the magnitude of $f_{b \text {, max }}(x)$ according to the equality of force, the width of the equivalent stress block can be calculated by 


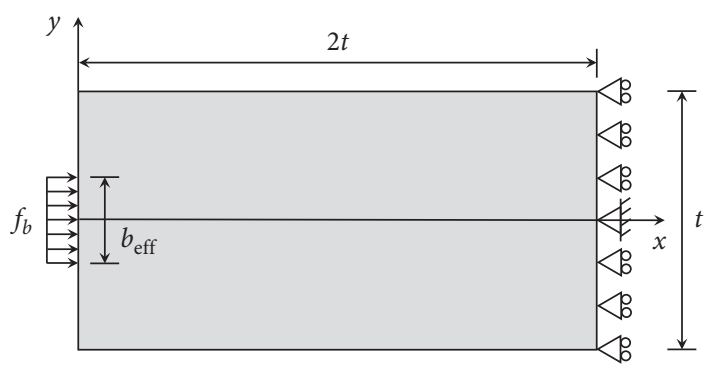

Figure 4: Structural analysis model for anchorage zone without a duct hole (plan view).

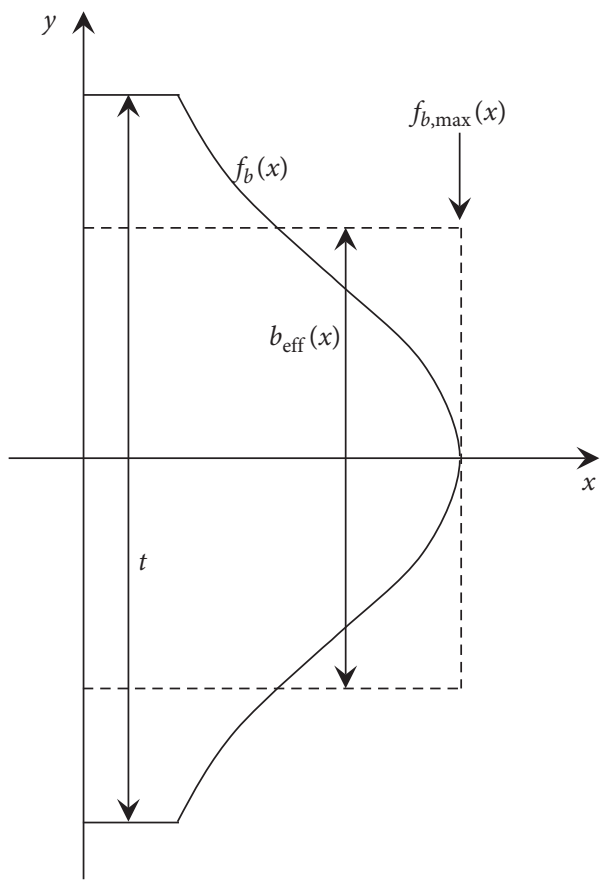

FIGURE 5: Longitudinal stress distribution at a section of an anchorage zone.

$b_{\text {eff }}(x)=\left(f_{b} / f_{b \text {, max }}(x)\right) b_{\text {eff }}$. By connecting the width $b_{\text {eff }}(x)$ calculated at each section along $x$, the curves that depict the spreading phenomenon of the longitudinal stress shown in Figure 2 can be obtained. $f_{c a}$ in equation (1), which corresponds to $f_{b, \max }(x)$ in this context, is based on this approximate curved shape of the spreading stress.

Figure 6 shows the contour of longitudinal stress for $b_{\text {eff }} / t=0.3$ as a representative analysis result. As previously expected, the length of the disturbed region in stress where the stress is not uniformly distributed is similar to the member thickness $t$. Table 1 presents a comparison between $f_{b, \max }(x)$ obtained by finite element method (FEM) and $f_{c a}$ calculated by equation (1) presented in AASHTO [2] at $x=$ $1.15 b_{\text {eff }}$ and midsection $(y=0)$. In equation (1), $f_{b}=P_{u} / A_{b}$, and the coefficient 0.6 and variable $\kappa$ (which were introduced to account for the effect of the vertical arrangement of anchorages) were not considered to ensure a reasonable comparison with $2 \mathrm{D} \mathrm{FE}$ analysis.

As shown in Table 1, the values obtained by FE analysis and the AASHTO predictive equation were very similar, and the difference only ranged from 1.8 to $4.1 \%$ in the case where the effect of a duct hole was not considered. Furthermore, the AASHTO equation slightly overestimated the stress, which is desirable to ensure a safer and conservative design. Consequently, it was revealed that the AASHTO approximate equation can be used for anchorage zone design with sufficient accuracy in the case of no duct hole. In reality, however, in a posttensioned PSC structure, a certain number of ducts are arranged. Therefore, investigation of whether or not the AASHTO equation is effective even in this actual case is discussed in the following section.

3.3. Case with a Duct Hole. The configuration of the case with a duct hole shown in Figure 7, including supports and loading condition, was modeled for FE analysis. The material properties of concrete and the size of the elements are the same as those for the case without a duct hole. Similar to the case without a duct hole, $b_{\text {eff }} / t$ varies in a practical range of $0.3,0.5$, and 0.7 according to the principle explained in equation (3). Also, the diameter of the duct $d$ was set for $d / b_{\text {eff }}$ to have the value of $0.5,0.6$, and 0.7 , which falls within 


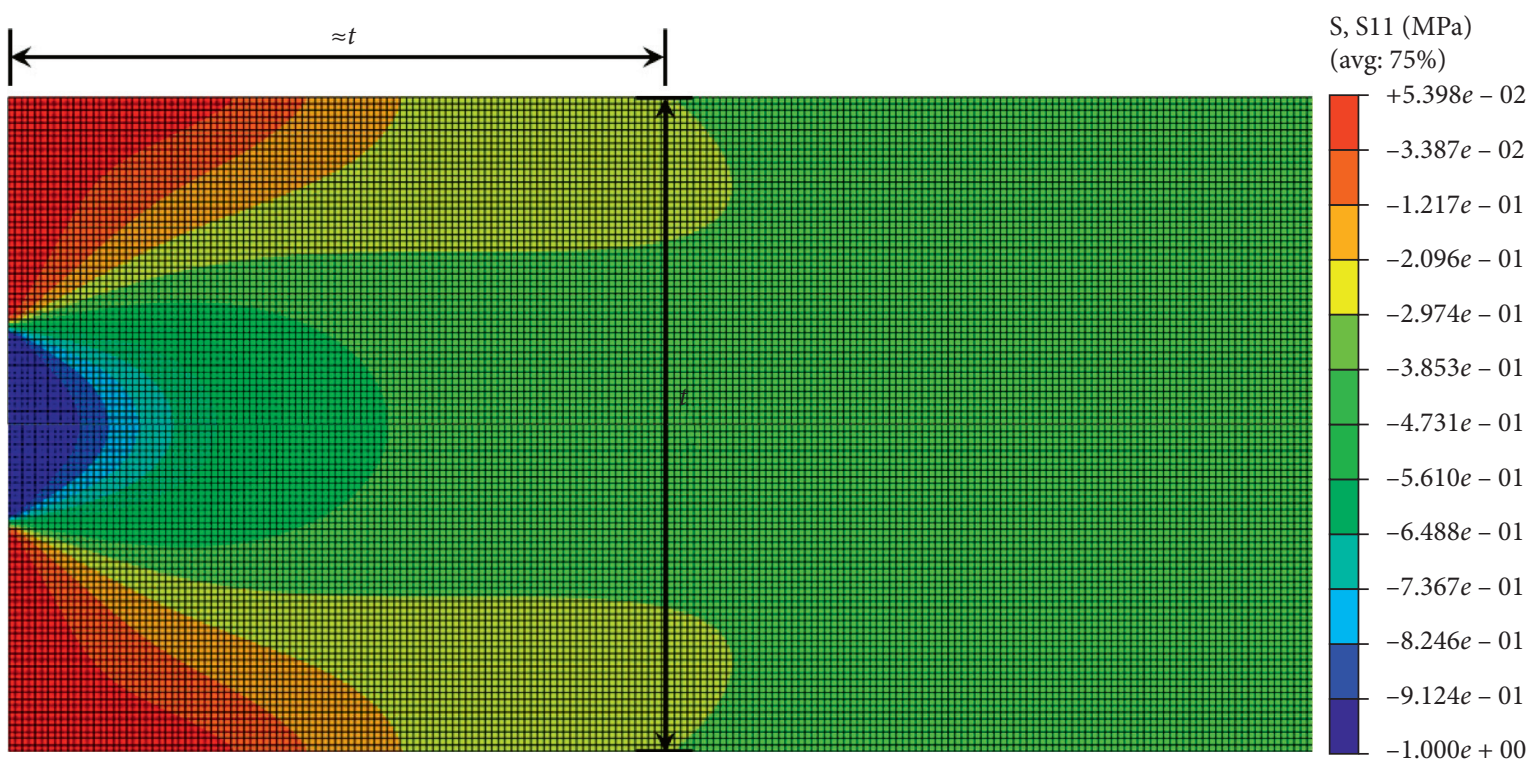

Figure 6: Contour of longitudinal stress (without a duct hole and $b_{\text {eff }} / t=0.3$ ).

TABLe 1: Comparison of longitudinal stress (without a duct hole, $x=1.15 b_{\text {eff }}$, and $y=0$ ).

\begin{tabular}{lccc}
\hline$b_{\text {eff }} / t$ & FEM $\left(f_{\left.b, \max , x=1.15 b_{\text {eff }} / f_{b}\right)}\right.$ & AASHTO $\left(f_{c a} / f_{b}\right)$ & AASHTO/FEM \\
\hline 0.3 & 0.536 & 0.554 & 1.034 \\
0.5 & 0.610 & 0.635 & 1.041 \\
0.7 & 0.730 & 0.743 & 1.018 \\
\hline
\end{tabular}

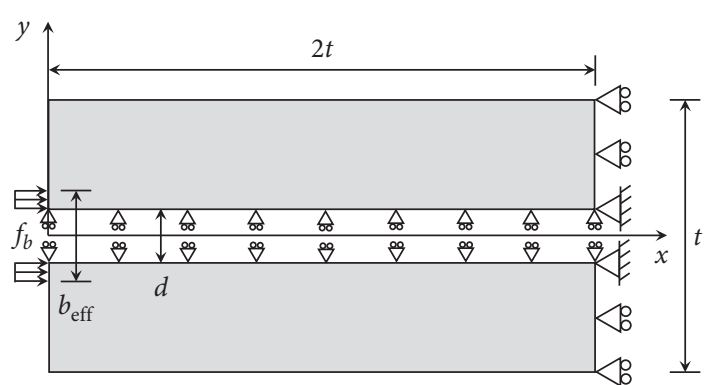

FIGURE 7: Structural analysis model for anchorage zone with a duct hole (plan view).

a practical range of $d / b_{\text {eff }}$. This value of $d / b_{\text {eff }}$, rather than the specific values of $d$ and $b_{\text {eff }}$, directly affects $f_{b}(x) / f_{b}$ that is the main concern of this study as shown in equation (3).

In Figure 7, the boundary condition at the duct hole and hole in anchorage device affects the stress distribution. Since the stiffness of the steel anchorage device is usually sufficient against deformation, the radial displacement at the inner surface of the hole which occurs during tensioning can be neglected. Although the stiffness of the duct is not as high as that of the anchorage device, it can also be assumed that the corrugated steel duct usually used has sufficient stiffness to resist and minimize the radial deformation. Therefore, a continuous roller support condition was applied to all the nodes located at the inner surface of the duct hole and hole in anchorage device, which restrains the displacements in the radial direction and allows displacements in the longitudinal direction. The discrete supports shown in Figure 7 were provided only for illustration.

Although the duct made of steel was not modeled in Figure 7, it was noted that a more detailed analysis by directly modeling the steel duct resulted in almost the same stress distribution. For example, $f_{c a} / f_{b}$ at the point of concern resulted in 0.311 in Table 2 for the case of $b_{\text {eff }} / t=0.5$ and $d / b_{\text {eff }}=0.6$ when the steel duct was not considered in FE analysis. On the other hand, when the steel duct was additionally incorporated into the FE model of Figure 7 using a plane stress element (CPS4R), the resulting $f_{c a} / f_{b}$ increased only by $2 \%$. The typical steel duct considered in the FE analysis had a thickness of $0.3 \mathrm{~mm}$, modulus of elasticity of $E_{s}=2 \times 10^{5} \mathrm{MPa}$ and Poisson's ratio of $v=0.30$. However, the corrugation of the duct was not modeled for simplification.

Figure 8 shows the contour of longitudinal stress for $b_{\text {eff }} / t=0.3$ and $d / b_{\text {eff }}=0.6$ as a representative analysis result. In the presence of the duct hole, the length of the disturbed region in stress was less than $t$ (unlike the case without a duct hole shown in Figure 6) and the assumption made in Figure 2 is therefore no longer valid. In this case, the length of the disturbed region appeared to be close to $t-d$, the reason for which is discussed below.

Table 2 presents a comparison between $f_{b \text {, max }}(x)$ obtained by FE analysis and $f_{c a}$ calculated by equation (1) at $x=1.15 b_{\text {eff }}$ and at the location of a duct $(y=d / 2)$. In equation (1), $f_{b}=P_{u} / A_{b}$, where $A_{b}$ is calculated by 
TABLE 2: Comparison of longitudinal stress (with a duct hole, $x=1.15 b_{\text {eff }}$, and $y=d / 2$ ).

\begin{tabular}{|c|c|c|c|c|c|c|}
\hline \multirow{2}{*}{$b_{\text {eff }} / t$} & \multirow{2}{*}{$d / b_{\text {eff }}$} & \multirow{2}{*}{$\operatorname{FEM}\left(f_{b, \max , x=1.15 b_{\mathrm{eff}}} / f_{b}\right)$} & \multicolumn{2}{|c|}{$\operatorname{AASHTO}\left(f_{c a} / f_{b}\right)$} & \multicolumn{2}{|c|}{ AASHTO/FEM } \\
\hline & & & Original equation (1) & Proposed equation (4) & Original equation (1) & Proposed equation (4) \\
\hline \multirow{3}{*}{0.3} & 0.5 & 0.298 & 0.554 & 0.346 & 1.859 & 1.161 \\
\hline & 0.6 & 0.243 & 0.554 & 0.289 & 2.280 & 1.189 \\
\hline & 0.7 & 0.185 & 0.554 & 0.227 & 2.995 & 1.227 \\
\hline \multirow{3}{*}{0.5} & 0.5 & 0.371 & 0.635 & 0.395 & 1.712 & 1.065 \\
\hline & 0.6 & 0.311 & 0.635 & 0.327 & 2.042 & 1.051 \\
\hline & 0.7 & 0.245 & 0.635 & 0.253 & 2.592 & 1.033 \\
\hline \multirow{3}{*}{0.7} & 0.5 & 0.539 & 0.743 & $0.485(0.538)^{*}$ & 1.378 & $0.900(0.998)^{*}$ \\
\hline & 0.6 & 0.482 & 0.743 & $0.402(0.482)^{*}$ & 1.541 & $0.834(1.0)^{*}$ \\
\hline & 0.7 & 0.411 & 0.743 & $0.307(0.411)^{*}$ & 1.808 & $0.747(1.0)^{*}$ \\
\hline
\end{tabular}

*Adjusted by equation (6b).

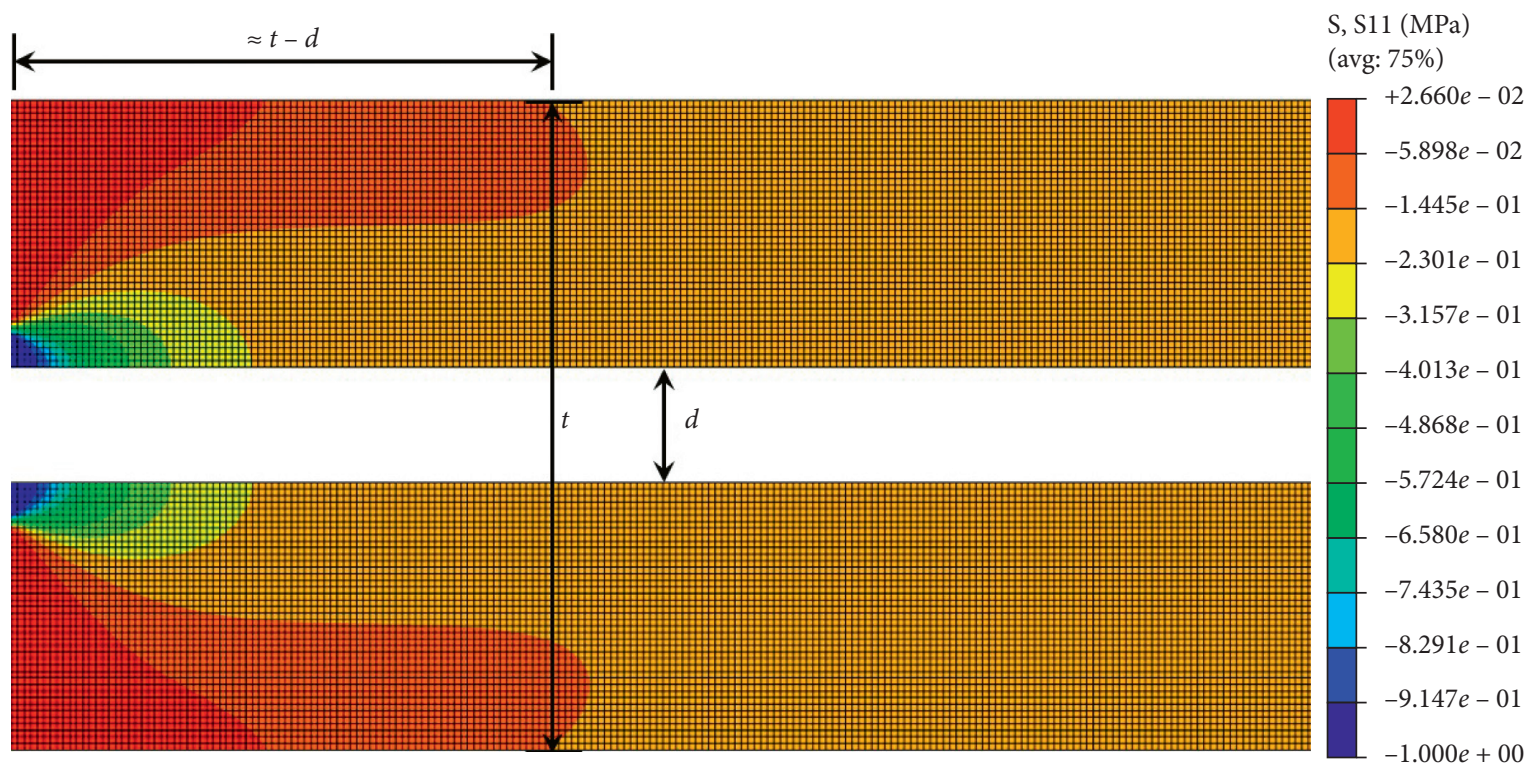

Figure 8: Contour of longitudinal stress (with a duct hole, $b_{\text {eff }} / t=0.3$, and $d / b_{\text {eff }}=0.6$ ).

excluding the area of the duct according to the definition of $A_{b}$. The coefficient 0.6 and variable $\kappa$ were also not considered for the abovementioned reason. As shown in Table 2, when the effect of the duct hole was taken into account, the AASHTO predictive equation considerably overestimated the stresses by $37.8 \sim 199.5 \%$ when compared to those obtained by FE analysis.

The primary reason for this unacceptably large difference between $f_{b, \max }(x)$ and $f_{c a}$ is the lack of proper consideration of the effect of the duct area on the stress distribution in equation (1) of AASHTO. That is, when the stress $f_{b}=$ $P_{u} / A_{b}$ was calculated, the duct area was deducted from $A_{b}$ according to the specification of AASHTO [2], which is definitely correct. However, the main problem of equation (1) is that the shape of the spread of stress is assumed to follow that of Figure 2, irrespective of the presence of the duct hole. As can be seen in the difference in the lengths of the disturbed region between Figures 6 and 8, the duct hole has a significant effect on the stress distribution. Nevertheless, equation (1) does not account for the difference incurred by the duct hole.
Therefore, it was identified that the stress may be largely overestimated, resulting in very inaccurate values when applying equation (1) for the design of an anchorage zone due to the presence of the duct hole. An improved procedure is proposed in the following chapter by incorporating the effect of the duct hole in the formula.

\section{Improvement of Design Equation for Anchorage Zone}

4.1. Proposal of Improved Equations. Judging from the stress distribution of the case with a duct hole shown in Figure 8, the distance at which the stress becomes constant is approximately $t-d$, which is shorter by the duct diameter $d$ than that in the case without a duct hole shown in Figure 6. In fact, this difference between the two cases can be expected based on the FE analysis results, the detailed explanation of which is given below. The structural behavior of the model shown in Figure 7 is symmetrical with respect to the center line of the duct because the configuration and the anchorage loading condition are symmetrical. Therefore, even if the 
model of Figure 7 is converted to that shown in Figure 9, the analysis results are identical. This implies that the analyses can also be performed using the alternative model with a reduced thickness of $t-d$ and without considering the duct hole, even though the duct hole exists in reality. In this model, the width subjected to the anchorage loading is also adjusted to $b_{\text {eff }}-d$ by deducting the duct diameter. It then follows that the length of the disturbed region becomes $t-d$ rather than $t$ because the member thickness is $t-d$ according to Saint-Venant's principle. This logic is demonstrated in the results shown in Figure 8.

Based on the above discussion, the AASHTO design equation shown in equation (1) needs to be modified to equation (4) by considering the effect of duct holes, by which a more realistic stress distribution can be obtained in the actual posttensioned PSC girders in which a certain number of ducts are installed. Other definitions of the notations are the same as those in equation (1), and the duct diameter $d$ was only added as a variable:

$$
f_{c a}=\frac{0.6 P_{u} \kappa}{A_{b}\left[1+l_{c}\left(\left(1 /\left(b_{\mathrm{eff}}-d\right)\right)-(1 /(t-d))\right)\right]} \leq 0.7 \phi f_{c i}^{\prime} .
$$

The stresses obtained using the improved equation (4) are presented in Table 2. Equation (4) gave values for $b_{\text {eff }} / t$ of 0.3 and 0.5 that were slightly larger than those of the FE analyses by $3.3 \sim 22.7 \%$ depending on the duct diameter. The appropriate level of safety and conservativeness were thus ensured through the proposed approximate design equation. On the contrary, the stresses obtained by equation (4) were smaller than those of FE analyses by $10.0 \sim 25.3 \%$ for $b_{\text {eff }} / t=$ 0.7 in a less safe manner for the following reason.

By introducing the parameters $\alpha$ and $\beta$ expressed as $b_{\text {eff }}=\alpha t$ and $d=\beta b_{\text {eff }}$, equation (5) can be derived for a distance of $1.15 b_{\text {eff }}$, where the stress is calculated:

$$
1.15 b_{\text {eff }}=\frac{1.15 \alpha}{1-\alpha \beta}(t-d) .
$$

For $\alpha=0.7$, that is, $b_{\text {eff }} / t=0.7$, the range of $1.15 b_{\text {eff }}$ in equation (5) becomes $1.239(t-d) \sim 1.578(t-d)$ for the range of $\beta=0.5 \sim 0.7$. Therefore, the distance $1.15 b_{\text {eff }}$ is located at the outside of the length of the disturbed region, $t-d$; equation (4) is thus no longer effective in this case. This problem was not observed in $b_{\text {eff }} / t=0.3$ or 0.5 . In fact, the original equation (1) of AASHTO is effective given that $l_{c}$ or $1.15 b_{\text {eff }}$ is located within the distance $t$ assumed for the disturbed region. The stress is almost uniformly distributed at locations deeper than $t$, while the stress estimated by equation (1) continuously decreases even when $l_{c}$ or $1.15 b_{\text {eff }}$ exceeds $t$. It can be presumed that this condition was not imposed in equation (1) because $l_{c}$ (less than $1.15 b_{\text {eff }}$ or $1.15 a_{\text {eff }}$ ) rarely exceeds $t$ in most cases.

Similarly, the proposed equation (4) can be effective under the condition that $l_{c}$ (less than $1.15 b_{\text {eff }}$ or $1.15 a_{\text {eff }}$ ) is smaller than $t-d$, while in the case of $l_{c}>t-d$, the stress should be maintained constant as the value at $l_{c}=t-d$. This case of $l_{c}>t-d$ would possibly occur more frequently in equation (4) than equation (1), because the length of the

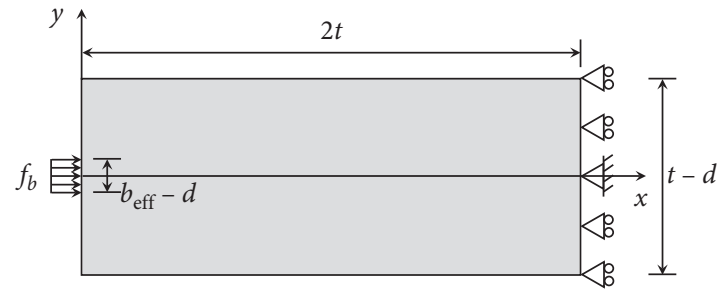

FIGURE 9: Alternative structural analysis model for anchorage zone with a duct hole (plan view).

disturbed region from equation (4) decreases due to the presence of the duct hole. Therefore, the proposed equation (4) can be further amended to equations (6a) and (6b) by dividing the range into two parts. It is shown in Table 2 that the stresses for $b_{\text {eff }} / t=0.7$ can be further adjusted to exact values by applying equation (6b):

$$
\begin{aligned}
f_{c a} & =\frac{0.6 P_{u} \kappa}{A_{b}\left[1+l_{c}\left(\left(1 /\left(b_{\mathrm{eff}}-d\right)\right)-(1 /(t-d))\right)\right]} \leq 0.7 \phi f_{c i}^{\prime}, \\
l_{c} & \leq t-d,
\end{aligned}
$$

$f_{c a}=\frac{0.6 P_{u} \kappa}{A_{b}}\left(\frac{b_{\mathrm{eff}}-d}{t-d}\right) \leq 0.7 \phi f_{c i}^{\prime}, \quad l_{c}>t-d$.

Figure 10 shows a comparison of the longitudinal stress distributions obtained by FE analyses and AASHTO equations for a representative case of $b_{\text {eff }} / t=0.3$ and $d / b_{\text {eff }}=$ 0.6 with a duct hole. In the AASHTO equations presented in equations (1), (6a), and (6b), variable $x$ was substituted for the original notation $l_{c}$ in order to plot the graphs. Note that the maximum longitudinal stress was denoted by $f_{b, \max }(x)$ in both FE analysis and the AASHTO equation in Figure 10. The meaning of $f_{b, \max }(x)$ and $f_{b}$ is as described in Section 3.2. The FE analysis results shown in Figure 10 verify that Saint-Venant's principle is effective, where the stresses approximately converge to a certain value at $x=t-d$ or $x=t$ for the cases with or without a duct hole, respectively, as was expected. The nondimensional converged value of $f_{b, \max }(x) / f_{b}$ can be readily estimated by the equilibrium of force and the area subjected to the stress: 0.146 from $\left(b_{\text {eff }}-\right.$ $d) /(t-d)$ or 0.3 from $b_{\text {eff }} / t$ for the cases with or without a duct hole, respectively. It is important to note that the original AASHTO equation intersects the curve for the FE analysis without a duct hole at $x=t$, and the comparison is only effective for $x<t$. On the other hand, the proposed improved AASHTO equation agrees with the curve for FE analysis with a duct hole at $x=t-d$ and they almost coincide for $x \geq t-d$ because equation (4) was further amended to equations (6a) and (6b).

As can be seen in Figure 10, the original equation (1) of AASHTO closely approximates the stress distribution for the case without a duct hole, but equation (1) cannot properly estimate the stress in the presence of the duct hole, resulting in considerably inaccurate values. Equations (6a) and (6b) can efficiently cope with these problems and show 


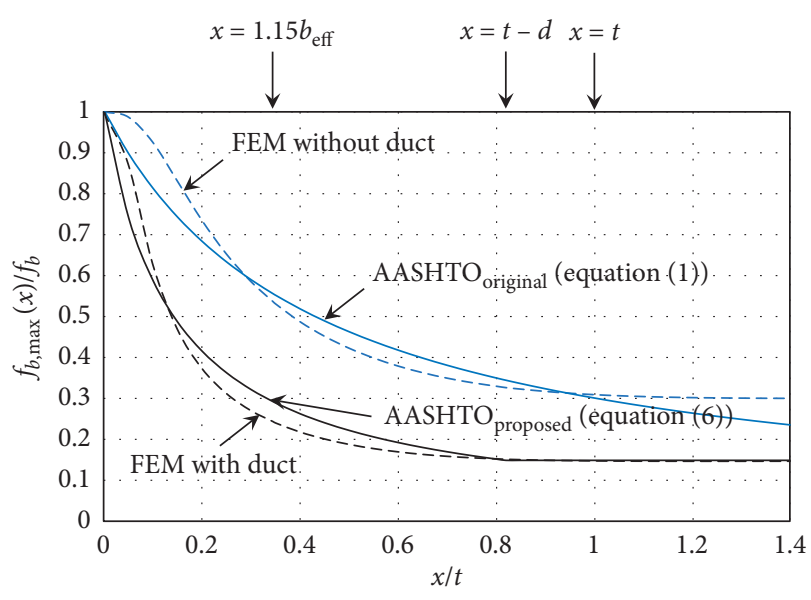

FIGURE 10: Comparison of longitudinal stress distribution in the cases with and without a duct hole $\left(b_{\text {eff }} / t=0.3\right.$ and $d / b_{\text {eff }}=0.6$ with a duct hole).

good agreement with the FE analysis results for the case with a duct hole.

4.2. Discussions. First, although Figure 10 shows that the stresses obtained by both AASHTO equations and FE analysis vary along the longitudinal direction in a curved shape, some difference in shape can be found between the two stress distributions. This can be attributed to the approximation made in equation (1) or equation (6a). Because the variation of the stress $f_{b \text {, max }}(x)$ is not linear along the $x$-axis, from the result shown in Figure 5, the spreading shape of the stress is also not strictly linear, although the shape is expressed as linear lines in Figure 2 for simplification. Provided that the FE analysis results more closely represent the actual situation, the corresponding AASHTO equation slightly overestimates or underestimates the stress depending on the location. In order to ensure the results of the AASHTO equation more closely approach those from the FE analysis, the form of the AASHTO equation can be modified, although it would be a complex yet trivial task from a practical point of view.

Secondly, the above FE analyses were performed in 2D problem to investigate structural behavior of the anchorage zone in transverse direction by considering the width and length of the member. The AASHTO equations were similarly analyzed focusing on the terms related to transverse structural behavior. However, the actual stress distribution at the $3 \mathrm{D}$ anchorage zone with pipe-shaped duct holes can differ slightly from that yielded by the AASHTO equations. In addition, although the diameters of the duct and the anchorage device are assumed to be the same along the length, in reality the hole diameter of the anchorage device slightly increases from the connection with the duct to the end. This is another minor discrepancy from the assumptions made in this study. It will be shown in chapter 5 that these assumptions are within an acceptable range when the results of numerical analysis are compared with those of the experiment.
Thirdly, the errors caused by using the original equation (1) of AASHTO instead of the proposed more accurate equation (4) can be examined by investigating the ratio of these two equations. Setting up $b_{\text {eff }}=\alpha t, d=\beta b_{\text {eff }}$, and $x=$ $\gamma b_{\text {eff }}$ by introducing the parameters $\alpha, \beta$, and $\gamma$, respectively, equation (7) can be established by manipulating the equations. This can be plotted as shown in Figure 11 for $\gamma=1.15$ which is the location considered. However, the discussion is effective for the condition of $(\gamma+\beta) b_{\text {eff }} \leq t$ because equation (7) is valid for $x \leq t-d$. Figure 11 shows that the errors significantly increase when $\beta$ (the ratio of duct diameter to lateral dimension of the anchor plate) is increased in the practical range of $\beta=0.5 \sim 0.7$. Also, a ratio greater than unity means that the stress can always be overestimated to a large extent if equation (1) is employed for the design, which may lead to an inaccurate and excessively conservative anchorage zone design. For example, if the stress calculated by equation (1) exceeds the allowable value of $0.7 \phi f_{c i}^{\prime}$ due to the overestimation, some conceivable countermeasures would include increasing $l_{c}$ or enlarging $a_{\text {eff }}$ or $b_{\text {eff }}$. These strategies can decrease the magnitude of the stress until equation (1) is satisfied by shifting the location for stress checking to the inner part of a member. However, they would cause uneconomical design because they require an increase of the amount of confining reinforcements or use of a larger-sized anchorage device which may actually be unnecessary according to the proposed equations (4), (6a), and (6b). It should be emphasized that the question raised in this study on the original equation (1) of AASHTO is related not simply to conservatism but rather to validity in the presence of the duct:

$$
\frac{\text { AASHTO }_{\text {original }}}{\text { AASHTO }_{\text {proposed }}}=\frac{1+\alpha \beta^{2}-(1+\alpha) \beta+(1-\alpha) \gamma}{(1-\beta)(1-\alpha \beta)[1+(1-\alpha) \gamma]} .
$$

\section{Comparison with Experimental Results}

In order to validate the accuracy of the proposed equation, the stress distributions, which were obtained by the original equation (1) of AASHTO and the improved AASHTO formula of equations (6a) and (6b) proposed in this study, were compared with that measured in the test of a posttensioned full-scale specimen [21, 22]. Figure 12 shows the specimen with the length, width, and thickness of $7 \mathrm{~m}, 4 \mathrm{~m}$, and $0.75 \mathrm{~m}$, respectively. Two ducts with $130 \mathrm{~mm}$ diameter were arranged with a center-to-center spacing of $2 \mathrm{~m}$ at midheight, where 31 strands with $15.2 \mathrm{~mm}$ diameter and ultimate tensile strength of 2,400 MPa were inserted in each duct. The test was originally performed to examine the appropriateness of the load transfer mechanism [23] in a PSC structure using high-strength strands [24]. The strands in each duct were tensioned up to $8,339 \mathrm{kN}$ using a multistrand jack at one end (live end), which was slightly over the prestressing force calculated based on maximum permissible stress of $0.80 f_{p u}$ and $0.94 f_{p y}$ [25], where $f_{p u}$ denotes the ultimate tensile strength and $f_{p y}$ represents the yield strength of strand. The specified compressive strength of the concrete was $40 \mathrm{MPa}$, and the strands were tensioned 


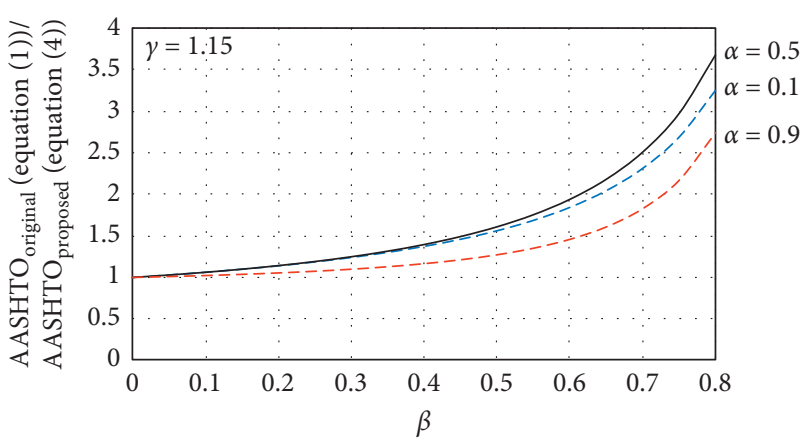

FIGURE 11: Comparison of errors between original and proposed AASHTO equations.

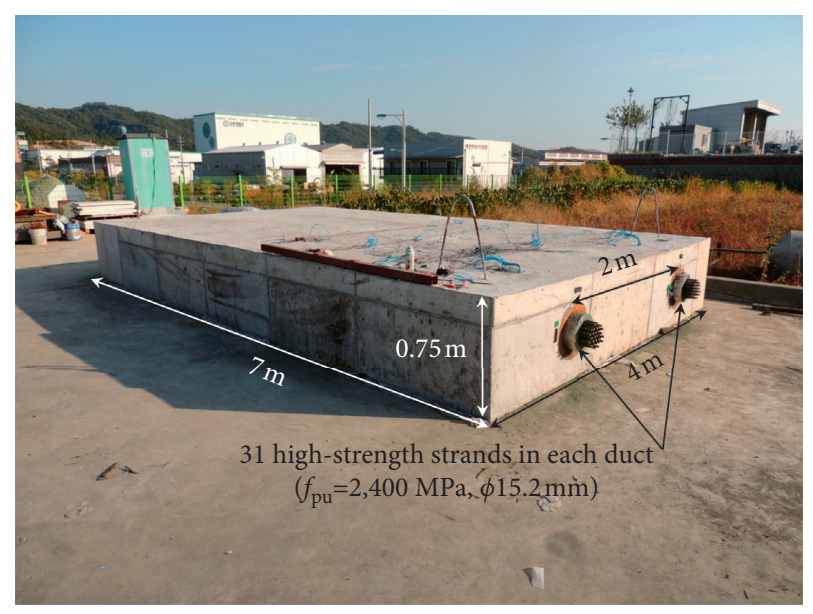

Figure 12: Full-scale test specimen.

when the concrete strength attained $28 \mathrm{MPa}$ which corresponds to $70 \%$ of the specified compressive strength. Spiral reinforcement was used as confining reinforcement of each anchorage zone according to the design procedure of ACI 318 [25]. The anchorage device was specially designed to resist a large amount of load exerted by high-strength strands [26].

Although a number of strain gauges were attached at the surface of concrete and steel, the data measured at the concrete embedment strain gauges were only analyzed for comparison with the theoretical values in this study. Figure 13 indicates the location of the embedment gauges in plan view that were embedded at midheight. The gauges were installed at a distance of $0.5,1.0,2.0$, and $3.0 \mathrm{~m}$ from the live end in the longitudinal direction and a total of 7 gauges were located in the transverse direction. The gauge was PMFL-60-5LT of electrical resistance type with a gauge length of $60 \mathrm{~mm}$. TDS-530 data logger was used to record the strains. The longitudinal compressive stresses induced in concrete during the tensioning operation were obtained by multiplying the measured strain with modulus of elasticity of concrete $\left(E_{c}=2.7 \times 10^{4} \mathrm{MPa}\right)$ which was calculated from the concrete strength [25].

Figure 14 compares the measured stresses with those calculated from two types of AASHTO equations in

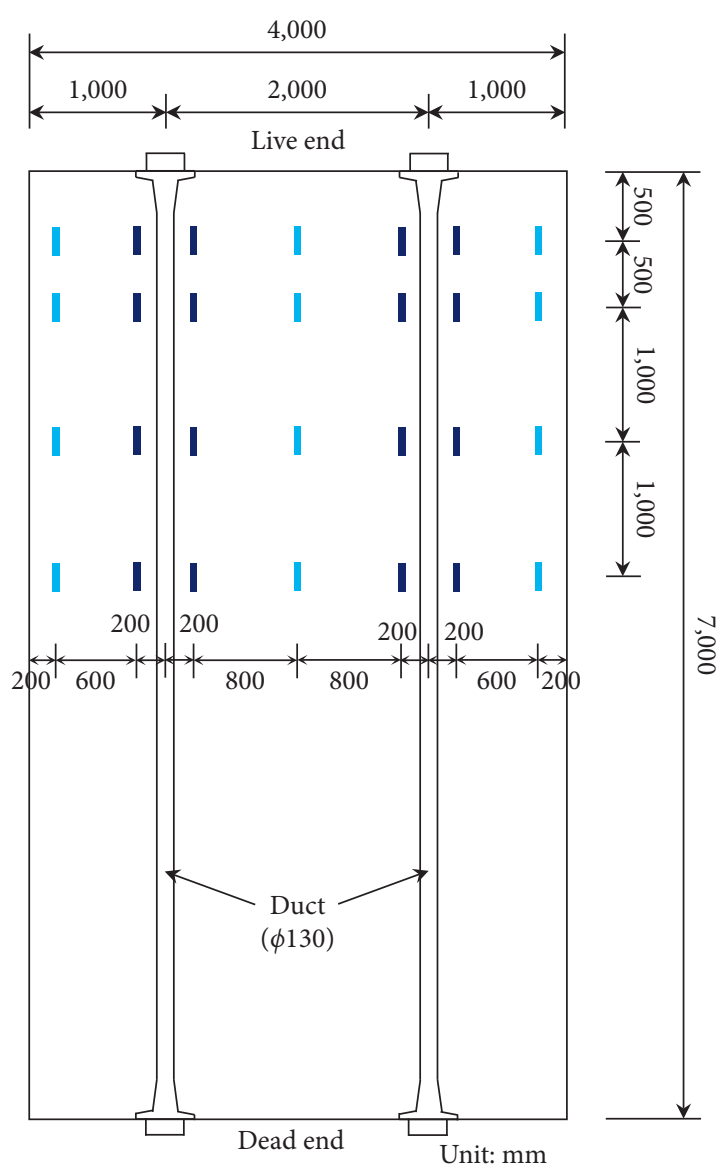

FIGURE 13: Location of concrete embedment strain gauges.

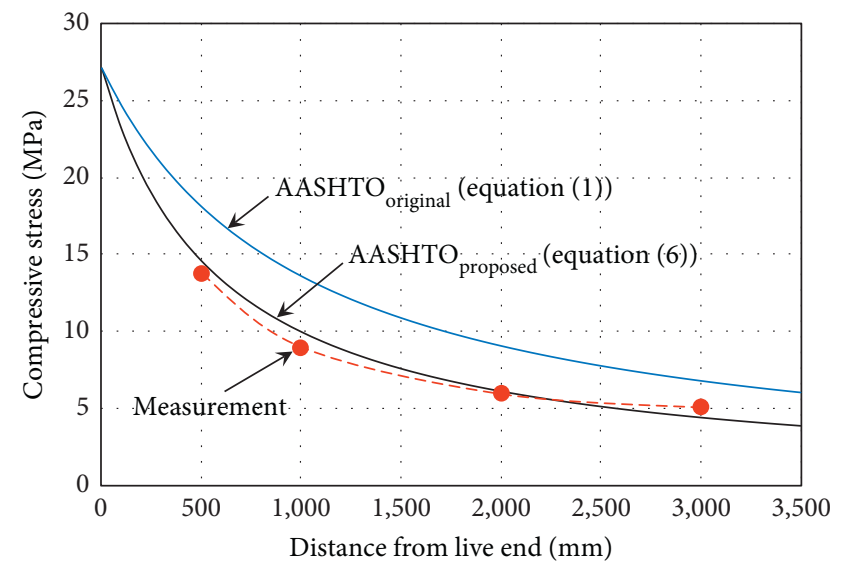

FIgURE 14: Comparison of longitudinal compressive stresses.

longitudinal direction. The values used for calculation are $t=750 \mathrm{~mm}, d=130 \mathrm{~mm}, \quad b_{\text {eff }}=430 \mathrm{~mm}$, and $P_{u}=8.339$ $\times 10^{6} \mathrm{~N}$. The stress measured at each longitudinal point was obtained by averaging the stresses measured near the ducts to reduce any bias. The gauges used for these stresses were marked by dark color in Figure 13. Although these stresses are slightly smaller than the maximum stress of interest occurring at the duct surface, the difference is not significant. It should be noted that the AASHTO equations shown in 
Figure 14 were used with the coefficient 0.6 to take into account the 3D stress distribution anticipated in the specimen. Also, the correction factor $\kappa$ in the equations can be regarded as unity because of $s \geq 2 b_{\text {eff }}$, which means the spacing of the anchorages is enough to avoid any interaction of the stress distribution [2]. Note that the horizontal direction of the specimen corresponds to the vertical direction in Figure 1(b).

It can be identified that the proposed AASHTO formula of equations (6a) and (6b) can estimate the actual stress distribution with sufficient accuracy. The difference between the measured value and equations (6a) and (6b) ranged only from 2.6 to $13.2 \%$. However, the original equation (1) of AASHTO overestimated the magnitude of stresses to a large extent by $31.3 \sim 52.7 \%$. These results apparently show how the stress distribution can reasonably be estimated by the proposed equation.

\section{Conclusions}

Among several design methods used for a posttensioned anchorage zone, a simplified approximate approach using the predictive equations specified in the AASHTO LRFD Bridge Design Specifications has been often used in practice. Validity of the equation used for predicting the compressive stress was investigated in this study among these equations. As a result, it was revealed that the current AASHTO equation can provide relatively accurate values for the case without a duct hole, whereas it can cause a significant error in the actual situation of a PSC structure with duct holes and can considerably overestimate the magnitude of the stresses in most cases. It was found through numerical analyses using the finite element method (FEM) and theoretical analyses such as Saint-Venant's principle that this discrepancy was caused because the effect of the duct holes on the stress distribution was not properly considered in the AASHTO equation. The difference in stress was as much as $37.8 \sim 199.5 \%$ when compared to the FE analysis results in the presence of the duct hole.

In order to cope with this problem, an improvement in the form of the original AASHTO equation was proposed in this study by accounting for the effect of the duct holes. The proposed equation showed good agreement with the actual distribution of the compressive stress induced at the anchorage zone by the prestressing tendons in the case with duct holes which was obtained analytically and experimentally. It also accurately predicted the magnitude of the stress at the location considered in the design of a special anchorage device. The difference from FE analysis results was considerably reduced to $-0.2 \sim 22.7 \%$ for the various cases considered in this study.

If the proposed equation is considered for possible revision of the anchorage zone design method specified in the AASHTO LRFD Bridge Design Specifications, the revised equation is expected to contribute to providing reasonably safe and economic design by preventing excessive arrangement of confining reinforcements or selection of an unnecessarily large anchorage device.

\section{Data Availability}

The data used to support the findings of this study are available from the author upon request.

\section{Conflicts of Interest}

The authors declare that there are no conflicts of interest regarding the publication of this paper.

\section{References}

[1] A. H. Nilson, Design of Prestressed Concrete, Wiley, New York, NY, USA, 2nd edition, 1987.

[2] American Association of State Highway and Transportation Officials (AASHTO), AASHTO LRFD Bridge Design Specifications, AASHTO, Washington, DC, USA, 8th edition, 2017.

[3] Korea Road and Transportation Association (KRTA), Design Code for Highway Bridges, KRTA, Seoul, Republic of Korea, 2010.

[4] D. H. Sanders and J. E. Breen, "Post-tensioned anchorage zones with single straight concentric anchorages," ACI Structural Journal, vol. 94, no. 2, pp. 146-158, 1997.

[5] Expressway and Transportation Technology Institute, "A study on improving arrangement of reinforcing bars in the anchorage zone of the PSC I girder," Korea Expressway Corporation, Hwaseong-si, Republic of Korea, EXTRI-201218-534.9607, 2011.

[6] Z.-Q. He and Z. Liu, "Investigation of bursting forces in anchorage zones: compression-dispersion models and unified design equation," Journal of Bridge Engineering, vol. 16, no. 6, pp. 820-827, 2011.

[7] L.-Y. Zhou, Z. Liu, and Z.-Q. He, "Further investigation of transverse stresses and bursting forces in post-tensioned anchorage zones," Structural Concrete, vol. 16, no. 1, pp. 84-92, 2015.

[8] A. Arab, S. S. Badie, M. T. Manzari, B. Khaleghi, S. J. Seguirant, and D. Chapman, "Analytical investigation and monitoring of end-zone reinforcement of the Alaskan Way viaduct super girders," PCI Journal, vol. 59, no. 2, pp. 109-128, 2014.

[9] Z. Ma, M. A. Saleh, and M. K. Tadros, "Optimized posttensioning anchorage in prestressed concrete I-beams," $P C I$ Journal, vol. 44, no. 2, pp. 56-73, 1999.

[10] P. Okumus and M. G. Oliva, "Evaluation of crack control methods for end zone cracking in prestressed concrete bridge girders," PCI Journal, vol. 58, no. 2, pp. 91-105, 2013.

[11] C. L. Roberts-Wollmann and J. E. Breen, "Design and test specifications for local tendon anchorage zones," ACI Structural Journal, vol. 97, no. 6, pp. 867-875, 2000.

[12] B. E. Ross, M. D. Willis, H. R. Hamilton, and G. R. Consolazio, "Comparison of details for controlling end-region cracks in precast, pretensioned concrete I-girders," PCI Journal, vol. 59, no. 2, pp. 96-108, 2014.

[13] C. Y. Tuan, S. A. Yehia, N. Jongpitaksseel, and M. K. Tadros, "End zone reinforcement for pretensioned concrete girders," PCI Journal, vol. 49, no. 3, pp. 68-82, 2004.

[14] J. W. Schmidt, A. Bennitz, B. Täljsten, P. Goltermann, and H. Pedersen, "Mechanical anchorage of FRP tendons-a literature review," Construction and Building Materials, vol. 32, pp. 110-121, 2012.

[15] J. Shi, X. Wang, Z. Wu, and Z. Zhu, "Effects of radial stress at anchor zone on tensile properties of basalt fiber-reinforced 
polymer tendons," Journal of Reinforced Plastics and Composites, vol. 34, no. 23, pp. 1937-1949, 2015.

[16] A. Al-Mayah, K. Soudki, and A. Plumtree, "Development and assessment of a new CFRP rod-anchor system for prestressed concrete," Applied Composite Materials, vol. 13, no. 5, pp. 321-334, 2006.

[17] J. E. Breen, O. Burdet, C. Roberts, D. Sanders, and G. Wollmann, "Anchorage zone reinforcement for posttensioned concrete girders," National Cooperative Highway Research Program (NCHRP) Report 356, Transportation Research Board, National Research Council, Washington, DC, USA, 1994.

[18] O. L. Burdet, Analysis and design of anchorage zones in posttensioned concrete bridges, The University of Texas at Austin, Austin, TX, USA, Ph.D. dissertation, 1990.

[19] S. P. Timoshenko and J. N. Goodier, Theory of Elasticity, McGraw-Hill, Columbus, OH, USA, 3rd edition, 1970.

[20] Dassault Systèmes Simulia Corporation, Abaqus 6.13-Analysis User's Manual, Dassault Systèmes Simulia Corporation, Providence, RI, USA, 2013.

[21] S.-J. Jeon, H.-K. Seo, J.-M. Yang, and S.-G. Youn, "Prestressing effect of LNG storage tank with 2,400 MPa highstrength strands," Journal of the Korean Society of Civil Engineers, vol. 36, no. 6, pp. 999-1010, 2016.

[22] Korea Concrete Institute (KCI), "Development of design guideline and analyses of LPG/LNG tank prestressed with 2400MPa PT," KCI, Seoul, Republic of Korea, KCI-R-15-018, 2015.

[23] European Organisation for Technical Assessment (EOTA), "Post-tensioning kits for prestressing of structures," EOTA, Brussels, Belgium, EAD 160004-00-0301, 2016.

[24] Korean Agency for Technology and Standards (KATS), Uncoated Stress-Relieved Steel Wires and Strands for Prestressed Concrete (KS D 7002), Korean Standards Association (KSA), Seoul, Republic of Korea, 2011.

[25] ACI Committee 318, Building Code Requirements for Structural Concrete (ACI 318-19), American Concrete Institute (ACI), Farmington Hills, MI, USA, 2019.

[26] Korea Total Anchorage Systems (KTA), Post-tensioning Systems of KTA, KTA, Gwangju-si, Republic of Korea, 2012. 